\title{
Phenyllactic acid: a potential antimicrobial compound in lactic acid bacteria
}

\begin{abstract}
3-Phenyllactic acid (PhLA) is a broad spectrum antimicrobial compound active against bacteria and fungi. PhLA exists in two chiral isomers, L-PhLA and D-PhLA which show antimicrobial activity. D-PhLA shows more antimicrobial activity than $\mathrm{L}-\mathrm{PhLA}$ and hence it is receiving great attention as food and feed additive in place antibiotics which control the microbial contamination and thereby increase the shelf life of food and food ingredients. This review summarizes the recent developments on the resources, detection and analysis and antimicrobial activity of PhLA. In addition, the article presents the recent studies of the key enzymes such as lactate dehydrogenases and pyruvate reductases involved in PhLA synthesis. The metabolic pathway and regulation of PLA synthesis in lactic acid bacteria (LAB) along with its high level production are also discussed.
\end{abstract}

Keywords: 3-phenyllactic acid, lactate dehydrogenase, antimicrobial activity, pyruvate reductase
Volume 2 Issue 5 - 2016

\author{
Chaudhari SS, Gokhale DV \\ CSIR-National Chemical laboratory, India
}

Correspondence: Gokhale DV, NCIM Resource Center, CSIR-National Chemical laboratory, Pune 4 I I008, India, Tel 912025902452, Fax 91202590267I, Email dv.gokhale@ncl.res.in

Received: August 19,2016 | Published: October 14,2016
Abbreviations: AAT, aromatic aminotransferase; GC/MS, gas chromatography/mass spectrometry; GRAS, generally accepted as safe; HPLC, high performance liquid chromatography; LDH, lactate dehydrogenase; MIC, minimum inhibitory concentration; NADH, nicotinamide adenine dinucleotide; NADPH, nicotinamide adenine phosphodinucleotide; PPA, phenylpyruvic acid; PhLA, phenyllactic acid; PGDH, d-3phosphoglycerate dehydrogenase

\section{Introduction}

3-Phenyl lactic acid (2-Hydroxy-3-Phenyl propionic acid, PhLA is an organic acid exhibiting broad spectrum antimicrobial activity against bacteria and fungi including yeast. ${ }^{1-3}$ Its molecular formula and molecular weight are $\mathrm{C} 9 \mathrm{H} 10 \mathrm{O} 3$ and $166 \mathrm{~g} / \mathrm{mol}$ respectively. PhLA exists in two chiral isomers namely D-PhLA and L-PhLA and both the isomers show antimicrobial activity. The antibacterial activity of PLA was first reported by Dieuleveux et al., ${ }^{4}$ in Geotrichum candidum. ${ }^{4}$ They purified PhLA from cultural broth of Geotrichum candidum was found to show anti-Listeria activity. They also found that $\mathrm{D}-\mathrm{PhLA}$ exhibited more anti-Listeria activity than L-PhLA. PhLA was also found to inhibit Gram positive and Gram negative bacteria and this effect was higher at acidic $\mathrm{pH}^{2}{ }^{2}$ The minimum inhibitory concentration (MIC) value of PhLA is relatively high $(50-100 \mathrm{mM}$ at $\mathrm{pH}$ 4-6) when it is used against yeast. ${ }^{3}$ The MIC value against fungi at $\mathrm{pH} 4.0$ is $45 \mathrm{mM}$ and it decreases with decrease in $\mathrm{pH} .{ }^{5}$ Though the mechanism of the antimicrobial action is not clear, it was shown by scanning electron microscopy that it damages the cell wall when the bacteria were exposed to PLA. It was observed that the bacteria form aggregates, secrete polysaccharides and loose cell wall rigidity leading to cell death.

Due to the antimicrobial property of PhLA, it is used in food industry as natural antimicrobial compound to control the fungal contaminations which help in extending the shelf life of food and food ingredients. It diffuses in to the food and feed very easily due to its hydrophilic nature. PhLA has been shown to exert immunemodulatory effects in poultry feed which improves the production performance and egg quality. ${ }^{7}$ It is known to show anti-pathogenic property in the large intestine when supplemented with chick feed diets which improves the meat quality. ${ }^{8}$ Such immune-modulatory and anti-pathogenic effects were observed in growing pigs when fed with diets supplemented with PhLA. ${ }^{9}$ It is noteworthy to mention that the analogue of PhLA known as "Danshensu" used in Chinese medicine is presently used for the treatment of coronary disease. ${ }^{10} \mathrm{PhLA}$ is marketed as skin-protecting agent, which reduce the skin wrinkles. ${ }^{11}$ $\mathrm{PhLA}$ and their derivatives also act as a chiral building block for the synthesis of many bio-based materials in agricultural, pharmaceutical and chemistry fields. ${ }^{12-14}$ It can be polymerized in to poly-PhLA which unlike polylactic acid showed high ultraviolet absorption ability and thermostability due to bulky aromatic side chain. ${ }^{15,16}$ The antimicrobial property, the promising applications of PhLA and its great demand sparked the great interest in the development of various strategies for its synthesis. Thus the bio-protective potential of PhLA producing LAB is high and well suited to wide applications in food safety and nutritional medicine. ${ }^{17,18}$

\section{PhLA occurrence and its detection}

PhLA was found in honey in higher amounts than other phenolic acids. PhLA content in thistle unifloral honey was around 100$800 \mathrm{mg} / \mathrm{kg}^{19}$ but the high concentrations of PLA were found in heather $(820 \mathrm{mg} / \mathrm{kg})$, ling heather $(875 \mathrm{mg} / \mathrm{kg})$ and manuka honeys $(243 \mathrm{mg} / \mathrm{kg}){ }^{20}$ Very recently, anti-staphylococcal activity against methicillin resistant Staphylococcus aureus was reported in manuka honey which contained high levels of acetic and lactic acids. ${ }^{21}$ It was found that $\mathrm{LAB}$ produced $\mathrm{PhLA}$ as a reaction product of amino acid metabolism. ${ }^{22,23}$ Its detection by reverse-phase HPLC is simple and widely adopted method for quantitative measurement of PhLA present in fermentation broth, ${ }^{24}$ honey ${ }^{19}$ and rumen fluid. ${ }^{25} \mathrm{GC} / \mathrm{MS}$ and capillary electrophoresis were also used for quantitative measurement of PLA. However, these methods could not detect the PhLA chiral isomers. PhLA enantiomers, L- and D-PhLA were determined by chiral HPLC method which involves the use of Chiracel OJ-RH column to separate PLA enantiomers and a UV detector at $205 \mathrm{~nm} .{ }^{26}$ 
The use of chiral additives such as modified cyclodextrins during chromatography and capillary electrophoresis methods could separate both the PhLA enantiomers. ${ }^{27}$

\section{Metabolic pathways and enzymes involved in pla synthesis}

PLA synthesis in LAB results from amino acid metabolism and the acids involved in PLA synthesis are phenylalanine and $\alpha$-ketoglutarate. The phenylalanine is first transaminated to phenylpyruvic acid (PPA) and PPA is further reduced to PhLA..$^{28-30}$ The transamination reaction is mediated by aromatic amino acid transferase (AAT) which has broad substrate specificity including leucine, tyrosine and methionine. ${ }^{31}$ The enzyme AAT transfer the amino acid group from phenyl alanine to any suitable ó-keto-acid preferably ó-ketoglutarate in LAB. Hence $\alpha$-ketoglutarate is an important that effects the both catabolism of phenylalanine and regulation of PhLA synthesis indirectly. ${ }^{32}$ This regulatory effect was observed in L. plantarum resulting in enhanced production of PLA in presence $\alpha$-ketoglutarate. ${ }^{28,30}$ In presence of substrates like glucose, citric acid and fructose acting as electron acceptor, glutamate dehydrogenase activity is enhanced leading to formation of $\alpha$-ketoglutarate which in turn upregulate the PhLA synthesis. ${ }^{32}$ The other precursor is PPA which can be reduced to PhLA with help of enzymes such as reductases or dehydrogenases present in LAB. When the Lactobacillus strains were grown in media supplemented with PPA in place of phenylalanine, PLA production was enhanced several fold indicating that PPA serves as better precursor than phenylalanine. ${ }^{28}$ The transamination of phenylalanine was a limiting factor which is a bottle-neck in PhLA synthesis. Hence it was suggested that the use of PPA could overcome this bottle-neck leading to increased PhLA synthesis. ${ }^{33,34}$

Different kinds of dehydrogenases have been reported to convert PPA to PhLA and the most reports suggest that lactate dehydrogenases (LDHs) are mainly responsible for PPA to PhLA. The other dehydrogenases especially, D-type dehydrogenases such as D-mandelate dehydrogenase (D-ManDH) from Enterococcus faecalis $^{35}$ and D-hydroxyisocaproate dehydrogenase (D-HicDH) from Lactobacillus casei $^{36}$ are also to involve in catalytic dehydrogenation. These D-type of enzymes exhibit broad substrate specificity including PPA. ${ }^{17}$ Fujii et al. ${ }^{37}$ discovered a novel fungal strain, Wickerhamia fluorescens TK1 which produced novel enzyme known as PPA reductase that reduced PPA to D-PhLA using either NADPH or NADH. ${ }^{37}$ This enzyme has more preference for NADPH than NADH. They demonstrated that this PPA reductase belongs to D-isomer specific 2-hydroxyacid dehydrogenase family. Very recently, novel NADH dependent PPA reductase was identified from Lactobacillus spp. CGMCC 9967 which belongs to D-3phosphoglycerate dehydrogenase (PGDH) subfamily of 2-hydroxy acid dehydrogenase superfamily. ${ }^{38}$ It is noteworthy to state that these PPA reductases prefer aromatic $\alpha$-keto acids than aliphatic keto acids and PPA was reduced more efficiently than other aromatic keto acids. In the synthesis of PhLA in LAB, PPA is converted to PhLA by LDH. There are two types of LDH existing in LAB based on catalytic specificity. PPA is converted to either L-PLA by L-LDH (EC.1.1.1.27) or D-PhLA by D-LDH (EC.1.1.1.28). These enzymes are optimally active at $\mathrm{pH}$ 5-7 and temperature ranging from $30^{\circ} \mathrm{C}$ to $45^{\circ} \mathrm{C}$. The $\mathrm{LAB}$ are mesophilc and acidophilic in nature and therefore the LDH in them exhibited less thermostability. LDH reported from B. coagulans and T. ethanolicus showed optimal activity and stability at higher temperatures. ${ }^{34,39}$ The possible pathway for PLA synthesis and enzymes involved in it are given in Figure 1.

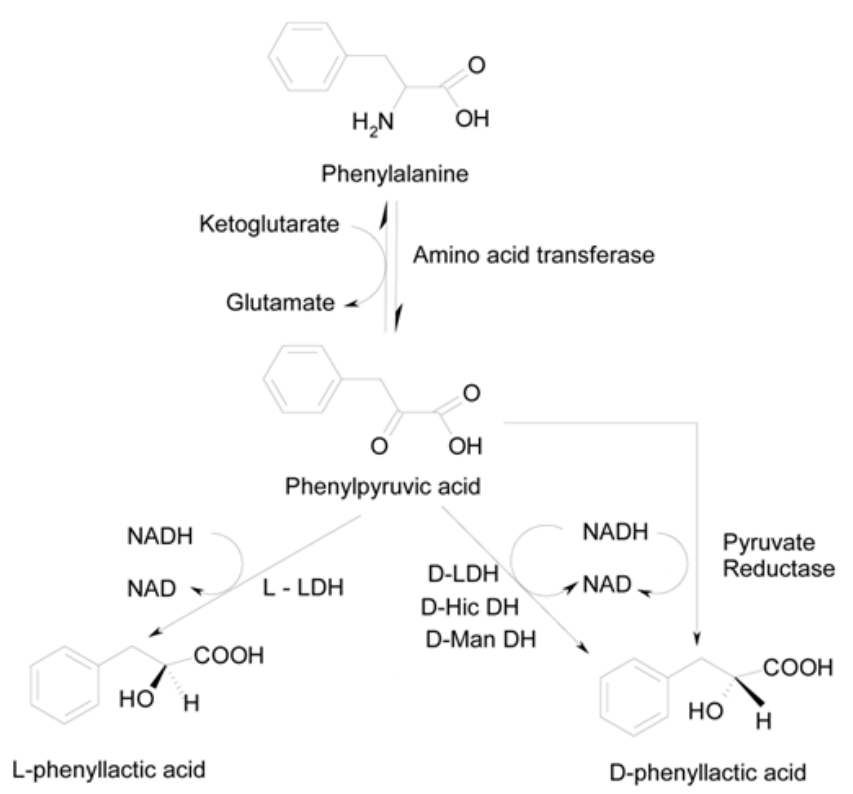

Figure I Possible pathways and enzymes involved in Phenyllactic acid production.

\section{Microorganisms producing pla and their im- provement}

PhLA is produced by wide range microorganisms including LAB, non-LABs and fungi such as Aspergillus, Geotrichum. ${ }^{17,29,33}$ Table 1 summarizes the production of PhLA by some of the promising bacteria which produced significant amounts of PhLA. It was found that $\mathrm{PhLA}$ is produced by various LAB species such as Lactobacillus, Leuconostoc and Enterococcus. Among the Lactobacillus species, L. plantarum, L. acidophilus, L. paracasei are reported to produce PhLA in significant quantities. We identified the L. lactis NCIM 5449 which produces high levels of D-lactic acid ${ }^{40}$ and it was also used for D-lactic acid production from sugarcane bagasse derived cellulose. ${ }^{41}$ The strain was found to produce $18 \mathrm{~g} / \mathrm{L}$ of D-PhLA from $25 \mathrm{~g} / \mathrm{L}$ of PPA within $2 \mathrm{~h}$ in biotransformation reaction (unpublished data). Arena et al., ${ }^{42}$ evaluated 79 wine derived L. plantarum strains for their antimicrobial activity against food borne pathogens..$^{42}$ They found that several L. plantarum strains showed significant ability to contrast various pathogenic bacteria which contaminate the food. Apart from bacteriocins produced by LAB that are effective against Grampositive bacteria, these strains are known to produce organic acids including PhLA and hydroxyl-PhLA which exhibit a broad spectrum of antimicrobial action. Among the non-LABs, Bacillus coagulans and Brevibacterium lactofermentum produced high amounts of PhLA. In general, PPA is converted to PhLA by LDHs present in LAB and therefore the studies were carried out on cloning and expression of native or site-mutated LDHs to enhance the PhLA productivity (Table 1). However, the attempts were not successful since there was no much increase in PhLA production. This could be attributed to low activity of native LDHs towards PPA due to its bulkier structure. An attempt was made to engineer D-LDH744 of Sporolactobacillus inulinus which resulted in increased enzymatic activities towards PPA using protein structure- guided modeling analysis. ${ }^{43}$ When this engineered D-LDHM307L was expressed in E.coli, the strain produced $21.43 \mathrm{~g} / \mathrm{L}$ of D-PhLA. This increase was due to enhanced enzymatic activity of the engineered enzyme towards bulkier PPA. Most of the studies on engineering D-LDH of $L$. pentosus dealt with the replacement of 
Tyr52 with such as Leucine, ${ }^{44}$ Val and Ala. ${ }^{45}$ Zhu et al. ${ }^{46}$ also replaced Tyr52 of D-LDH of L. pentosus with small hydrophobic residues to enhance the PhLA synthesis activity. ${ }^{46}$ Zheng et al., ${ }^{47}$ selected Tyr52 and Phe299 of D-LDH of L. pentosus for site directed mutagenesis to reduce steric exclusion effect. ${ }^{47}$ However, only Y52L mutant of D-LDH gave good performance and showed elevated activities towards unnatural substrates with large substitutes at C-3 position. The gene responsible for PPA reductase from Lactobacillus sp.
CGMCC 9967 was co-expressed with glucose dehydrogenase from Bacillus megaterium in E. coli ${ }^{38}$ for efficient D-PhLA production with internal NADPH regeneration system. The newly constructed $E$. coli converted $100 \mathrm{~g} / \mathrm{L}$ of PPA to PhLA with $91.3 \%$ isolation yield and $99 \%$ ee and the productivity was about $10 \mathrm{~g} / \mathrm{L} / \mathrm{h}$. To our knowledge, no traditional microbial strain improvement strategies such as mutations are reported which give the enhanced yields and productivity of PLA.

Table I Production of PLA by some promising organisms including genetically engineered strains

\begin{tabular}{|c|c|c|c|c|c|}
\hline Strains & Production process & Chirality & Pla (g/l) & Yield (\%) & Reference \\
\hline Lactobacillus plantarum IMAU 10124 & Fermentation & Racemic & 2.9 & 96 & 48 \\
\hline Lactobacillus sp. SK007 & Biotransformation & Racemic & 1.13 & 56.7 & 28 \\
\hline Lactobacillus sp. SK007 & Fed-batch fermentation & Racemic & 17.38 & 51.1 & 33 \\
\hline Lactobacillus lactis NCIM 5449 & Biotransformation & D-PLA & 18.5 & 74 & Our studies (unpublished data) \\
\hline Bacillus coagulans & Biotransformation & Racemic & 37.3 & 70 & Zheng et al. $^{34}$ \\
\hline Leuconostoc mesenteroides ATCC 8293 & Biotransformation & D-PLA & 5.82 & 75.2 & 49 \\
\hline Brevibacterium lactofermentum & Fermentation & D-PLA & 1.92 & -- & 50 \\
\hline E. Coli BL2I (DE3) & Biotransformation & D-PLA & 15.6 & 77 & 46 \\
\hline E. Coli JMI09 (DE3) & Biotransformation & D-PLA & 21.4 & 82.3 & 43 \\
\hline E. Coli BL2I (DE3) with pET28-gdh-T7-ppr & Biotransformation & D-PLA & 91.3 & 91.3 & 38 \\
\hline
\end{tabular}

\section{Future perspective}

To our knowledge, there is limited information available on biological production of PhLA although natural foods such as honey and $\mathrm{LAB}$ fermented foods contain PLA in significant amounts. However, the use of PhLA as a pure compound is not permitted in food since no studies carried out on its metabolism in the human body and toxicity effects. The generation of this data is essential to make PhLA GRAS cleared. Also No literature is available on its recovery from the cultural or biotransformation broth which is the prerequisite for total integrated process for PhLA synthesis followed by its recovery in pure form. PhLA was produced by adding phenylalanine or PPA in the fermentation medium. The fermentation broth contains several compounds which make the recovery of pure PhLA cumbersome. The alternative approach would be the biotransformation in which whole cells are used to convert PPA to PhLA. Biotransformation is usually carried out in presence of glucose and fructose needed for cofactor (NADH/NADPH) regeneration. The biotransformation broth does not contain much of the impurities and hence the downstream processing for recovery of PhLA would be simpler and easy. Lactobacillus strains are fastidious and hence they need rich medium for their growth which may not be cost effective. We suggest that the Lactobacillus cells generated during D-lactic acid production may be used to convert PPA to PhLA using biotransformation approach. This will save the cost of growing the Lactobacillus strains separately. ${ }^{48-50}$

\section{Acknowledgements}

None.

\section{Conflict of interest}

The author declares no conflict of interest.

\section{References}

1. Lavermicocca P, Valerio F, Visconti A. Antifungal activity of phenyllactic acid against molds isolated from bakery products. Appl Environ Microbiol. 2003;69(1):634-640.

2. Ohhira I, Kuwaki S, Morita H, et al. Identification of 3-phenyllactic acidas a possible antimicrobial substance produced by Enterococcus faecalis TH10. Biocontrol Sci. 2004;9(3):77-81.

3. Schwenninger SM, Lacroix C, Truttmann S, et al. Characterization of low molecular weight anti-yeast metabolites produced by a foodprotective Lactobacillus-Propionobacterrium coculture. J Food Prot. 2008;71(12):2481-2487.

4. Dieuleveux V, Van der Pyl D, Chataud J, et al. Purification and characterization of anti-Listeria compounds produced by Geotrichum candidum. Appl Environ Microbiol. 1998;64(2):800-803.

5. Strom K, Sjogren J, Broberg A, et al. Lactobacillus plantarum MiLAB 393 produces the antifungal cyclic dipeptides cyclo (L-Phe-L-Pro) and cyclo (L-Phe-trans-4-OH-LPro) and 3-phenyllactic acid. Appl Environ Microbiol. 2002;68(9):4322-4327.

6. Dieuleveux V, Lemarinier S, Gueguen M. Antimicrobial spectrum and target site of D-3-phenyllactic acid. Int J Food Microbiol. 1998;40(3):177-183.

7. Wang JP, Yoo JS, Lee JH, et al. Effects of phenyllactic acid on production performance, egg quality parameters, and blood characteristics in laying hens. J Appl Poultyr Res. 2009;18:203-209.

8. Wang JP, Lee JH, Yoo JS, et al. Effects of phenyllactic acid on growth performance, intestinal microbiota, relative organ weight, blood characteristics, and meat quality of broiler chicks. Poultry Sci. 2010;89(7):1549-1555.

9. Wang JP, Yoo JS, Lee JH, et al. Effects of phenyllactic acid on growth performance, nutrient digestibility, microbial shedding, and blood profile in pigs. J Anim Sci. 2009;87(10):3235-3243. 
10. Wang J, Shao Y, Zhang Y, et al. Experimental studies on $\beta$-phenyllactic acid on the coronary system. J Shanghai Med Univ. 1991;18:295-297.

11. Yu R, Van Scott E. Method of using 3-phenyllactic acid for treating wrinkles. Patent US 5643953.

12. Adachi Y, Kimura K, Saigusa M, et al. Growth promotion of rice (Oryza Sativa L.) seedlings by application of $\mathrm{L}-\beta$-phenyllactic acid. Asian $J$ Plant Sci. 2014;8:64-71.

13. Ebdrup S, Pettersson I, Rasmusson HB, et al. Synthesis and biological structural characterization of the duel-acting peroxisome proliferator-activated receptor $\alpha / \gamma$ agonist ragaglitazar. J Med Chem. 2013;46(8):1306-1317.

14. Sato K, Ito H, Ei H. Microbial conversion of phenyllactic acid to Lphenylalanine. Patent JP 86212293. 1986.

15. Fujita T, Nguyen HD, Ito T, et al. Microbial monomers customsynthesized to build true bio-derived aromatic polymers. Appli Microbiol Biotechnol. 2013;97(20):8887-8894.

16. Kawaguchi H, Uematsu K, Ogino C, et al. Simultaneous and fermentation of kraft pulp by recombinant $E$. coli for phenyllactic acid production. Biochem Eng J. 2014;88:188-194.

17. $\mathrm{Mu} \mathrm{W}, \mathrm{Yu} \mathrm{S}$, Zhu L, et al. Recent research on 3-phenyllactic acid, a broad-spectrum antimicrobial compound. Appl Microbiol Biotechnol. 2012;95(5):1155-1163.

18. Pawlowska AM, Zannini E, CoffeyA, et al. Green preservatives: combating fungi in the food and feed industry by applying antifungal lactic acid bacteria. Adv Food Nutr Res. 2012;66:217-238.

19. Tuberoso CI, Bifulco E, Caboni P, et al. Lumichrome and phenyllactic acid as chemical markers of thistle (Galactites tomentosa Moench) honey. J Agri Food Chem. 2011;59(1):364-369.

20. Dimitrova B, Gevrenova R, Anklam E. Analysis of phenolic acids in honeys of different floral origin by solid-phase extraction and high performance liquid chromatography. Phytochem Anal. 2007;18(1):24 32.

21. Mannina L, Sobolov AP, Coppo E, et al. Anti-staphylococcal activity and metabolite profiling of manuka honey (Leptospermum scoparium L.) after in vitro simulated digestion. Food Funct. 2016;7(3):1664-1670.

22. Van der Muelen R, Scheirlinck I, Van Schoor A, et al. Population dynamics and metabolite target analysis of lactic acid bacteria during laboratory fermentations of wheat and spelt sourdougs. Appl Environ Microbiol. 2007;73(15):4741-4750.

23. Ryan LA, Dal Bello F, Czemy M, et al. Quantification of phenyllactic acid in wheat sourdough using high resolution gas chromatographymass spectrometry. J Agric Food Chem . 2009;57(3):1060-1064.

24. Armaforte E, Carri S, Ferri G, et al. High performance liquid chromatography determination of phenyllactic acid in MRS broth. $J$ chromatogr A. 2006;1131(1-2):281-284.

25. Khan RI, Amin MR, Mohammed N, et al. Quantitative determination of aromatic amino acids and related compounds in rumen fluid by highperformance liquid chromatography. J Chromatogr B Biomed Sci Appl. 1998;710(1-2):17-25.

26. Tekewe A, Singh S, Singh M, et al. Development and validation of HPLC method for the resolution of drug intermediates: DL-3-phenyllactic acid, DL-O-acetyl-3-phenyllactic acid and (+/-)-mexiletine acetamide enantiomers. Talanta. 2008;75(1):239-245.

27. Heil M, Podebrad F, Beck T, et al. Enantio-selective multidimensional gas chromatography-mass spectrometry in the analysis of urinary organic acids. J Chromatogr B Biomed Sci Appl. 1998;714(2):119-126.

28. Li XF, Jiang B, Pan BL. Biotransformation of phenylpyruvic acid to phenyllactic acid by growing and resting cells of a Lactobacillus sp. Biotechnol Lett. 2007;29(4):593-597.
29. Valerio F, Lavermicocca P, Pascale M, et al. Production of phenyllactic acid by lactic acid bacteria: an approach to the selection of strains contributing to food quality and preservation. FEMS Microbiol Lett. 2004;233(2):289-295.

30. Vermeulen N, Ganzle MG, Vogel RF. Influence of peptide supply and cosubstrates on phenylalanine metabolism of Lactobacillus sanfranciscensis DSM2045(T) and Lactobacillus plantarum TMW1.468. J Agrc Food Chem. 2006;54(11):3832-3839.

31. Yvon M, Thirouin S, Rijnen L, et al. An aminotransferase from Lactobacillus lactis initiates conversion of amino acids to cheese flavor compounds. Appl Environ Microbiol. 1997;63(2):414-419.

32. Dallagnol AM, Catalan CAN, Mercado MI, et al. Effect of biosynthetic intermediates and citrate on the phenyllactic and hydroxyphenyllactic acids production by Lactobacillus plantarum CRL 778. J Appl Microbiol. 2011;111:1447-1455.

33. Mu MW, Liu FL, Jia JH, et al. Optimization of cultural medium for the production of phenyllactic acid by Lactobacillus $s p$. SK007. Bioresour Technol. 2009;100(3):1366-1370.

34. Zheng ZJ, Ma CQ, Gao C, et al. Efficient conversion of phenylpyruvic acid to phenyllactic acid by using whole cells of Bacillus coagulans SDM. PloS one. 2011;6(4):e19030.

35. Wada Y, Iwai S, Tamura Y, et al. A new family of D-2-hydroxyacid dehydrogenases that comprises D-mandelate dehydrogenases and 2ketopantoate reductases. Biosci Biotechnol Biochem. 2008;72(4):10871094.

36. Hummel W, Schutte H, Kula MR. D-2-hydroxyisocaproate dehydrogenase from Lactobacillus casei - a new enzyme suitable for stereospecific reduction of 2-ketocarboxylic acids. Appl Microbiol Biotechnol. 1985;21:7-15.

37. Fujii T, Shimizu M, Doi Y, et al. Novel fungal phenylpyruvate reductase belongs to D-isomer specific 2-hydroxyacid dehydrogenase family. Biochim Biophys Acta. 2011;1814(12):1669-1676.

38. Xu GC, Zhang LL, Ni Y. Enzymatic preparation of D-phenyllactic acid at high space-time yield with a novel phenylpyruvate reductase identified from Lactobacillus sp. CGMCC 9967. J Biotechnol. 2016;222:29-37.

39. Zhou Q, Shao WL. Molecular genetic characterization of the thermostable L-lactate dehydrogenase gene (ldhL) of Thermoanaerobacter ethanolicus JW200 and biochemical characterization of the enzyme. Biochem (Mosc). 2010;75(4):526-530.

40. Joshi DS, Singhvi MS, Khire JM, et al. Strain improvement of Lactobacillus lactis NCIM 2368 for D-lactic acid production. Biotechnol Lett. 2010;32(4):517-520.

41. Singhvi M, Joshi D, Adsul MG, et al. D(-) lactic acid production from cellobiose and cellulose by Lactobacillus lactis mutant RM2-24. Green Chem. 2010;12:1106-1109.

42. Arena MP, Silvain A, Normanno G, et al. Use of Lactobacillus plantarum strains as a biocontrol strategy against food-borne pathogenic microorganisms. Frontiers Microbiol. 2016;7:464.

43. Wang M, Zhu L, Xu X, et al. Efficient production of enantiomerically pure D-phenylactate from phenylpyruvate by structure-guided design of an engineered D-lactate dehydrogenase. Appl Microbiol Biotechnol. 2016;100(17):7471-7478.

44. Tokuda C, Ishikura Y, Shigematsu M, et al. Conversion of Lactobacillus pentosus D-lactate dehydrogenase through a single amino acid replacement. J Bacteriol. 2003;185(16):5023-5026.

45. Ishikura Y, Tsuzuki S, Takahashi O, et al. Recognition site for the side chain of 2-ketoacid substrate in D-lactate dehydrogenase. J Biochem. 2005;138(6):741-749.

46. Zhu Y, Hu F, Zhu Y, et al. Enhancement of phenyllactic acid biosynthesis by recognition site replacement of D-lactate dehydrogenase from Lactobacillus pentosus. Biotechnol Lett. 2015;37(6):1233-1241. 
47. Zheng Z, Sheng B, Gao C, et al. Higly stereoselective biosynthesis of (R)-á-hydroxycarboxylis acids through rationally redesigned mutation of D-lactate dehydrogenase. Sci Rep. 2013;3:3401.

48. Zhang X, Zhang S, Shi Y, et al. A new high phenyllactic acid-yielding Lactobacillus plantarum IMAU10124 and a comparative analysis of lactate dehydrogenase gene. FEMS Microbiol Lett. 2014;356(1):89-96.
49. Li L, Shin SY, Lee KW, et al. Production of natural antimicrobial compound D-phenyllactic acidusing Leuconostoc mesenteroides ATCC 8293 whole cells involving highly active D-lactate dehydrogenase. Lett Appl Microbiol. 2014;59(4):404-411.

50. Kamata M, Toyomasu R, Suzuki D, et al. D-phenylactic acid production by Brevibacterium or Corynebacterium. Patent JP 86108396. 